RESEARCH ARTICLE

\title{
Asymmetric pulse effects on pair production in polarized electric fields
}

\author{
Obulkasim Olugh ${ }^{1,2}, \mathrm{Zi}$-Liang $\mathrm{Li}^{3}$, and Bai-Song Xie ${ }^{1,4}$ \\ ${ }^{1}$ Key Laboratory of Beam Technology of the Ministry of Education, and College of Nuclear Science and Technology, Beijing Normal \\ University, Beijing 100875, China \\ ${ }^{2}$ Xinjiang Police College, Urumqi 830011, China \\ ${ }^{3}$ School of Science, China University of Mining and Technology, Beijing 100083, China \\ ${ }^{4}$ Beijing Radiation Center, Beijing 100875, China \\ (Received 1 July 2020; revised 6 August 2020; accepted 31 August 2020)
}

\begin{abstract}
Using the Dirac-Heisenberg-Wigner formalism, effects of the asymmetric pulse shape on the generation of electronpositron pairs in three typical polarized fields, i.e., linear, middle elliptical and circular fields, are investigated. Two kinds of asymmetries for the falling pulse length, short and elongated, are studied. We find that the interference effect disappears with the shorter pulse length and that the peak value of the momentum spectrum is concentrated in the center of the momentum space. In the case of the extending falling pulse length, a multiring structure without interference appears in the momentum spectrum. Research results show that the momentum spectrum is very sensitive to the asymmetry of the pulse as well as to the polarization of the fields. We also find that the number density of electronpositron pairs under different polarizations is sensitive to the asymmetry of the electric field. For the short falling pulse, the number density can be significantly enhanced by over two orders of magnitude. These results could be useful in planning high-power and/or high-intensity laser experiments.
\end{abstract}

Keywords: asymmetric pulse; electron-positron pair production; polarized fields

\section{Introduction}

In intense electromagnetic fields the vacuum state is unstable and spontaneously generates electron-positron $\left(e^{-} e^{+}\right)$ pairs. This is known as the Schwinger effect, which is one of the highly nontrivial predictions in quantum electrodynamics $(\mathrm{QED})^{[1-3]}$. Because of the tunneling nature of the Schwinger effect, this interesting phenomenon is exponentially suppressed and the pair production rate is proportional to $\exp \left(-\pi E_{\mathrm{cr}} / E\right)$, where the corresponding Schwinger critical field strength $E_{\mathrm{cr}}=m_{e}^{2} c^{3} / e \hbar=1.3 \times$ $10^{18} \mathrm{~V} / \mathrm{m}$. The associated laser intensity, e.g., $I=4.3 \times$ $10^{29} \mathrm{~W} / \mathrm{cm}^{2}$, is too high and beyond current technological possibilities. Its detection has therefore remained a challenge for many decades ${ }^{[4]}$. However, current advances in highpower laser technology ${ }^{[5-7]}$ and forthcoming experimental

Correspondence to: B.-S. Xie, College of Nuclear Science and Technology, Beijing Normal University, Beijing 100875, China. Email: bsxie@bnu.edu.cn facilities (for example, the Extreme Light Infrastructure, the Exawatt Center for Extreme Light Studies, and the Station of Extreme Light at the Shanghai Coherent Light Source) have led to the hope of QED predictions entering the realm of observation. On the other hand, using X-ray free-electron laser facilities can in principle yield a strong field at about $E=0.1 E_{\mathrm{cr}}=1.3 \times 10^{17} \mathrm{~V} / \mathrm{m}^{[8]}$ and drive interest in studying pair production under super strong fields.

The Schwinger effect is one of the nonperturbative phenomena in QED, while the understanding of it is still far from complete. Therefore, studying pair production in the nonperturbative regime would deepen our knowledge about the relatively less tested branch of QED. Motivated by this, many exploratory studies of the Schwinger effect based on a number of different theoretical techniques have been undertaken, for example, within the quantum kinetic approach $^{[9-11]}$ and the real time Dirac-Heisenberg-Wigner (DHW) formalism ${ }^{[12-18]}$, the WKB approximation ${ }^{[19,20]}$ as well as the worldline instanton technique ${ }^{[21]}$. Schützhold et $a l .{ }^{[21]}$ found that the pair production rate can be strongly 
enhanced by superimposing the slowly varying strong field with the rapid oscillating weak field, which is now called the dynamically assisted Schwinger effect. In Ref. [22], by using the quantum kinetic approach, the momentum spectrum of the produced pairs has been computed, and the spectrum was found to be extremely sensitive to these physical pulse parameters. The pair production in a pulsed electric field ${ }^{[23]}$ is to present the signature of the effective mass of the created particles in the strong oscillating electric field. In Refs. [2426] researchers have shown the importance of pulse shape effects on the pair creation process in different situations. For a concrete description of the various approaches and relevant publications, see our review of pair production ${ }^{[27]}$.

In this paper we further investigate the Schwinger effect by considering the asymmetric pulse shape with Gaussian envelope and different polarizations. We mainly consider asymmetric pulse shape effects on pair production in different polarizations, e.g., linear, elliptic and circular polarizations. We reveal some novel features of the momentum spectra of created pairs for differently polarized electric fields. In this study the real-time DHW formalism is employed as it leads to efficient calculations in the case of a circularly ${ }^{[28-30]}$ or elliptically polarized electric field ${ }^{[31,32]}$.

This paper is organized as follows. In Section 2 we introduce the model of a background field. In Section 3 we briefly introduce the DHW formalism that is used in our calculations for completeness. In Section 4 we show the numerical results for momentum spectra and analyze the underlying physics. In Section 5 we give the numerical results for the pair number density. We end the paper with a brief summary and discussion in Section 6.

\section{External electric field model}

We focus on the study of $e^{-} e^{+}$pair production in differently polarized and time-dependent asymmetric electric fields. The explicit form of the external field is given as

$$
\begin{aligned}
\mathbf{E}(t)= & \frac{E_{0}}{\sqrt{1+\delta^{2}}}\left[e^{-\left(t / \tau_{1}\right)^{2} / 2} \theta(-t)+e^{-\left(t / \tau_{2}\right)^{2} / 2} \theta(t)\right] \\
& \times\left(\begin{array}{c}
\cos (\omega t+\phi) \\
\delta \sin (\omega t+\phi) \\
0
\end{array}\right),
\end{aligned}
$$

where $E_{0} / \sqrt{1+\delta^{2}}$ is the field amplitude, $\tau_{1}$ and $\tau_{2}$ are the rising and falling pulse durations, respectively, $\theta(t)$ is the Heaviside step function, $\omega$ is the oscillation frequency, $\phi$ is the carrier phase and $\delta$ represents the field polarization (or the ellipticity). The field parameters are chosen as $E_{0}=$ $0.1 \sqrt{2} E_{\mathrm{cr}}, \omega=0.6 m, \tau_{1}=10 / m$ and $\phi=0$, where $m$ is the electron mass. For the falling pulse length, we set the parameter as $\tau_{2}=k \tau_{1}$, where $k$ is the ratio of the falling to rising pulse length. Throughout this paper, we use natural units $\hbar=c=1$.
The main interest in this study is asymmetric pulse duration effects on pair production in differently polarized and time-dependent asymmetric electric fields. We mainly consider two different situations when the rising pulse length $\tau_{1}$ is fixed. One in which the falling pulse length $\tau_{2}=k \tau_{1}$ becomes shorter with $0<k \leq 1$, and another in which the falling pulse length $\tau_{2}=k \tau_{1}$ becomes longer with $k \geq 1$.

\section{Brief outline of the DHW formalism}

The DHW formalism is an approach used to describe the quantum phenomena of a system by a Wigner function as the relativistic phase space distribution. It has also been further adopted in the studies of Sauter-Schwinger QED vacuum pair production ${ }^{[12-16]}$. The DHW formalism automatically combines quantum electrodynamics with notions familiar from statistical physics ${ }^{[19,22]}$, and it allows one to incorporate temporal as well as spatial inhomogeneities ${ }^{[12-18]}$. Most importantly, the DHW formalism gives access to the relativistic phase-space distribution of the produced particles. In the following, we present a brief outline of the DHW formalism for completeness.

A convenient starting point is the gauge-invariant density operator of two Dirac field operators in the Heisenberg picture

$$
\hat{\mathcal{C}}_{\alpha \beta}(r, s)=\mathcal{U}(A, r, s)\left[\bar{\psi}_{\beta}(r-s / 2), \psi_{\alpha}(r+s / 2)\right],
$$

where $\psi_{\alpha}(x)$ is the electron's spinor-valued Dirac field, and $r$ and $s$ are the center-of-mass and relative coordinates, respectively. The Wilson-line factor before the commutators

$$
\mathcal{U}(A, r, s)=\exp \left[i e s \int_{-1 / 2}^{1 / 2} \mathrm{~d} \xi A(r+\xi s)\right]
$$

is used to keep the density operator gauge invariant, and this factor depends on the elementary charge $e$ and the background gauge field $A$. In addition, we use a meanfield (Hartree) approximation by replacing the gauge field operator with the background field.

The important quantity in the DHW method is the covariant Wigner operator, given as the Fourier transform of the density operator (Equation (2)):

$$
\hat{\mathcal{W}}_{\alpha \beta}(r, p)=\frac{1}{2} \int \mathrm{d}^{4} s e^{i p s} \hat{\mathcal{C}}_{\alpha \beta}(r, s)
$$

By taking the vacuum expectation value of the Wigner operator, we obtain the Wigner function

$$
\mathbb{W}(r, p)=\langle\Phi|\hat{\mathcal{W}}(r, p)| \Phi\rangle
$$

For numerical convenience, the Wigner function can be decomposed into a complete basis set $\left\{\mathbb{1}, \gamma_{5}, \gamma^{\mu}, \gamma_{5} \gamma^{\mu}, \sigma^{\mu \nu}\right.$ 
$\left.:=i\left[\gamma^{\mu}, \gamma^{\nu}\right] / 2\right\}$. Then we obtain the 16 covariant real Wigner components

$$
\mathbb{W}=\frac{1}{4}\left(\mathbb{1} \mathbb{S}+i \gamma_{5} \mathbb{P}+\gamma^{\mu} \mathbb{V}_{\mu}+\gamma^{\mu} \gamma_{5} \mathbb{A}_{\mu}+\sigma^{\mu \nu} \mathbb{T}_{\mu \nu}\right)
$$

Here $\mathbb{S}, \mathbb{P}, \mathbb{V}_{\mu}, \mathbb{A}_{\mu}$ and $\mathbb{T}_{\mu \nu}$ are scalar, pseudoscalar, vector, axial vector and tensor, respectively. According to Refs. [12-16] and by using the equations of motion for the fermionic Heisenberg operators, the dynamical equation for the Wigner function is

$$
D_{t} \mathbb{W}=-\frac{1}{2} \mathbf{D}_{\mathbf{x}}\left[\gamma^{0} \gamma, \mathbb{W}\right]+i m\left[\gamma^{0}, \mathbb{W}\right]-i \mathbf{P}\left\{\gamma^{0} \gamma, \mathbb{W}\right\}
$$

where $D_{t}, \mathbf{D}_{\mathbf{x}}$ and $\mathbf{P}$ denote the pseudodifferential operators

$$
\begin{gathered}
D_{t}=\partial_{t}+e \int_{-1 / 2}^{1 / 2} \mathrm{~d} \lambda \mathbf{E}\left(\mathbf{x}+i \lambda \nabla_{\mathbf{p}}, t\right) \times \nabla_{\mathbf{p}}, \\
\mathbf{D}_{\mathbf{x}}=\nabla_{\mathbf{x}}+e \int_{-1 / 2}^{1 / 2} \mathrm{~d} \lambda \mathbf{B}\left(\mathbf{x}+i \lambda \nabla_{\mathbf{p}}, t\right) \times \nabla_{\mathbf{p}}, \\
\mathbf{P}=\mathbf{p}-i e \int_{-1 / 2}^{1 / 2} \mathrm{~d} \lambda \lambda \mathbf{B}\left(\mathbf{x}+i \lambda \nabla_{\mathbf{p}}, t\right) \times \nabla_{\mathbf{p}} .
\end{gathered}
$$

Inserting decomposition (Equation (6)) into the equation of motion, Equation (7), for the Wigner function, we obtain a set of partial differential equations (PDEs) for the 16 Wigner components. Furthermore, for spatially homogeneous electric fields like Equation (1), by using the characteristic method $^{[28,29]}$ and replacing the kinetic momentum $\mathbf{p}$ with the canonical momentum $\mathbf{q}$ via $\mathbf{q}-e \mathbf{A}(t)$, the PDEs for the 16 Wigner components can be reduced to 10 ordinary differential equations of the nonvanishing Wigner coefficients

$$
w=\left(\mathbb{s}, \mathbb{V}_{i}, \mathrm{a}_{i}, \mathbb{t}_{i}\right), \quad \mathbb{t}_{i}:=\mathbb{t}_{0 i}-\mathbb{t}_{i 0} .
$$

For detailed derivations and explicit forms of these 10 equations, we refer the reader to Refs. [16, 17, 33]. Note that the corresponding vacuum nonvanishing initial values are

$$
\mathbb{S}_{\mathrm{vac}}=\frac{-2 m}{\sqrt{\mathbf{p}^{2}+m^{2}}}, \quad \mathbb{v}_{i, \mathrm{vac}}=\frac{-2 p_{i}}{\sqrt{\mathbf{p}^{2}+m^{2}}} .
$$

In the following, we express the scalar Wigner coefficient by the one-particle momentum distribution function

$$
f(\mathbf{q}, t)=\frac{1}{2 \Omega(\mathbf{q}, t)}\left(\varepsilon-\varepsilon_{\mathrm{vac}}\right),
$$

where $\Omega(\mathbf{q}, t)=\sqrt{\mathbf{p}^{2}(t)+m^{2}}=\sqrt{m^{2}+[\mathbf{q}-e \mathbf{A}(t)]^{2}}$ is the total energy of the electrons (positrons) and $\varepsilon=m s+$ $p_{i} \mathbb{\mathbb { V }}_{i}$ is the phase-space energy density. To obtain the oneparticle momentum distribution function $f(\mathbf{q}, t)$, referring to Refs. [28, 29], it is helpful to introduce an auxiliary threedimensional vector

$$
v_{i}[\mathbf{q}, t]:=\mathbb{V}_{i}[\mathbf{p}(t), t]-[1-f(\mathbf{q}, t)] \mathbb{V}_{i, \operatorname{vac}}[\mathbf{p}(t), t]
$$

So the one-particle momentum distribution function $f(\mathbf{q}, t)$ can be obtained by solving the following ordinary differential equations, including it as well as the other nine auxiliary quantities:

$$
\dot{f}=\frac{e \mathbf{E} \cdot \mathbf{v}}{2 \Omega}
$$

$$
\begin{gathered}
\dot{\mathbf{v}}=\frac{2}{\Omega^{3}}\left[(e \mathbf{E} \cdot \mathbf{p}) \mathbf{p}-e \mathbf{E} \Omega^{2}\right](f-1) \\
-\frac{(e \mathbf{E} \cdot \mathbf{v}) \mathbf{p}}{\Omega^{2}}-2 \mathbf{p} \times \mathbf{a}-2 m \mathbf{t}, \\
\dot{\mathbf{a}}=-2 \mathbf{p} \times \mathbf{v}, \\
\dot{\mathbf{t}}=\frac{2}{m}\left[m^{2} \mathbf{v}-(\mathbf{p} \cdot \mathbf{v}) \mathbf{p}\right],
\end{gathered}
$$

with the initial conditions $f(\mathbf{q},-\infty)=0, \mathbf{v}(\mathbf{q},-\infty)=$ $\mathbf{a}(\mathbf{q},-\infty)=\mathbf{t}(\mathbf{q},-\infty)=0$. Here the time derivative is indicated by a dot, $\mathbf{a}(\mathbf{q}, t)$ and $\mathbf{t}(\mathbf{q}, t)$ are the threedimensional vectors corresponding to Wigner components and $\mathbf{A}(t)$ denotes the vector potential of the external field.

Finally, by integrating the distribution function $f(\mathbf{q}, t)$ over the full momentum space, we obtain the number density of created pairs defined at asymptotic times $t \rightarrow+\infty$ :

$$
n=\lim _{t \rightarrow+\infty} \int \frac{\mathrm{d}^{3} q}{(2 \pi)^{3}} f(\mathbf{q}, t) .
$$

\section{Momentum spectra of the produced particles}

In this section we report some interesting results for the momentum spectra of the produced particles with several pulse parameters under typical cases of the polarization field, such as linear $(\delta=0)$, elliptical $(\delta=0.5)$ and circular $(\delta=1)$ fields.

\subsection{Linear polarization, $\delta=0$}

First, in Figure 1 we show the momentum spectra for different $k$ when we keep the rising pulse length $\tau_{1}$ fixed but change the falling pulse length $\tau_{2}=k \tau_{1}$ to be shorter with $0<k \leq 1$. For $k=1$, the momentum spectrum is centered at the origin, and weak oscillation is observed, as shown in the upper-left panel of Figure 1. The physical origin of the oscillation is explained in Ref. [20] in terms of the 

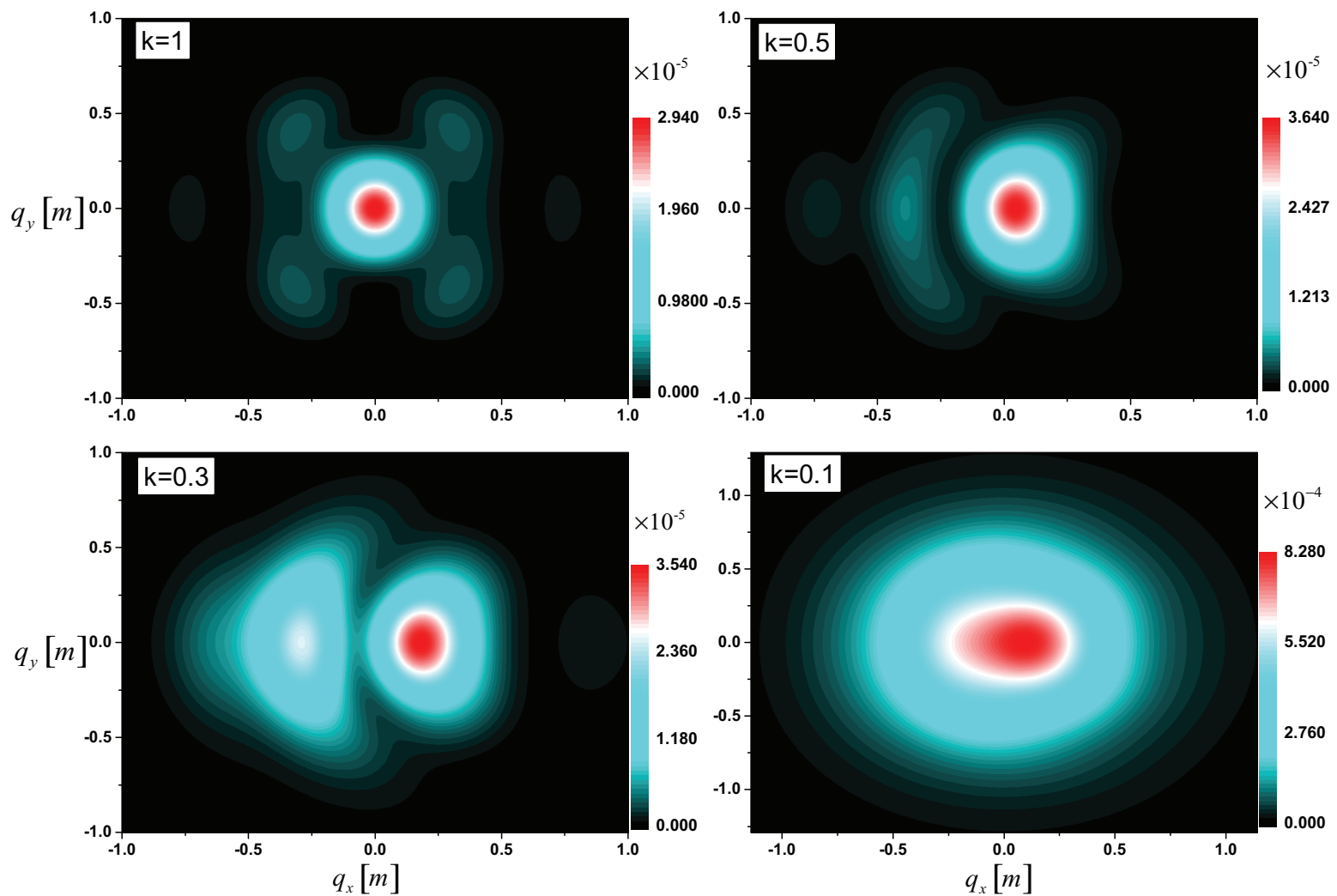

Figure 1. Momentum spectra of produced $e^{+} e^{-}$pairs for linear polarization $(\delta=0)$ at $q_{z}=0$ in the $\left(q_{x}, q_{y}\right)$ plane when the rising pulse length $\tau_{1}$ is fixed but the falling pulse length $\tau_{2}=k \tau_{1}$ becomes shorter with $0<k \leq 1$. The chosen parameters are $E_{0}=0.1 \sqrt{2} E_{\text {cr }}, \omega=0.6 m$ and $\tau_{1}=10 / m$, where $m$ is the electron mass.

interference between separate complex conjugate pairs of turning points.

It can be seen that the momentum spectrum of the created pair is very sensitive to the asymmetry of the electric field. When the ratio parameter $k$ is changed to $k=0.5$, the main peak of the momentum spectrum is shifted to the positive $q_{x}$ and the symmetry distribution of the momentum spectrum is destroyed. This effect is similar to the effect of carrier phase studied in Ref. [22]. Considering this fact that, for small $k$, pulse asymmetry plays the carrier-phase-like role, the physical explanation of momentum spectrum distribution due to pulse asymmetry can be understood by assuming that particles are created with vanishing initial longitudinal momentum. In the presence of $\mathbf{E}(t)$, the produced pairs are continuously accelerated, and particle momentum is mainly determined by its creation time ${ }^{[11,18]}$. At the earlier time $t_{0}$ it is created; after $t_{0}$, it has to be accelerated at the longer time and finally it gets the higher longitudinal momentum. In general, most pairs are expected to appear at those times corresponding to the local maxima of the field. Then those produced pairs are subject to acceleration by the electric field, and the gained momenta $\operatorname{are}^{[32]}$

$$
q=\int_{t_{0}}^{t} e \mathbf{E}(t) \mathrm{d} t=e \mathbf{A}\left(t_{0}\right)-e \mathbf{A}(t)
$$

Because of the fact that the vector potential vanishes at asymptotic times $t \rightarrow \infty$, the final particle momentum solely depends on the vector potential at the time when the particle was created. For example, the peak in Figure 1 when $k=1$ at $\mathbf{q}\left(t_{0}\right)=0$ is due to the dominant peak in the electric field at $t=0$. As $\mathbf{E}(t)$ decreases at later times, less particles are produced. However, as $\mathbf{A}(t)$ increases at the same time, these particles are effectively accelerated more strongly. So the peak positions and/or momentum spectrum patterns depend on the pulse shape. Furthermore, when $k=0.3$, the main parts of the momentum spectrum also appear for negative $q_{x}$ beside the positive $q_{x}$ peak, splitting the momentum spectrum. Therefore, two peaks are observed. This result is similar to the effect introduced by the frequencies chirp in Ref. [33]. For the very asymmetric case of $k=0.1$, the momentum spectrum of the particle is again concentrated in the center but the oscillation of the momentum spectrum disappears. Finally, we note that the peak value of the momentum spectrum of the pairs is increased from $2.94 \times 10^{-5}(k=1)$ to $8.28 \times 10^{-4}(k=0.1)$.

Second, in Figure 2 we show the momentum spectra for different $k$ when the rising pulse length $\tau_{1}$ is fixed but the falling pulse length $\tau_{2}$ becomes longer with $k \geq 1$. From this figure we can see that, as the field asymmetry increases, the main center peak of the momentum spectrum decreases while some disconnected ringlike structures with peaks 

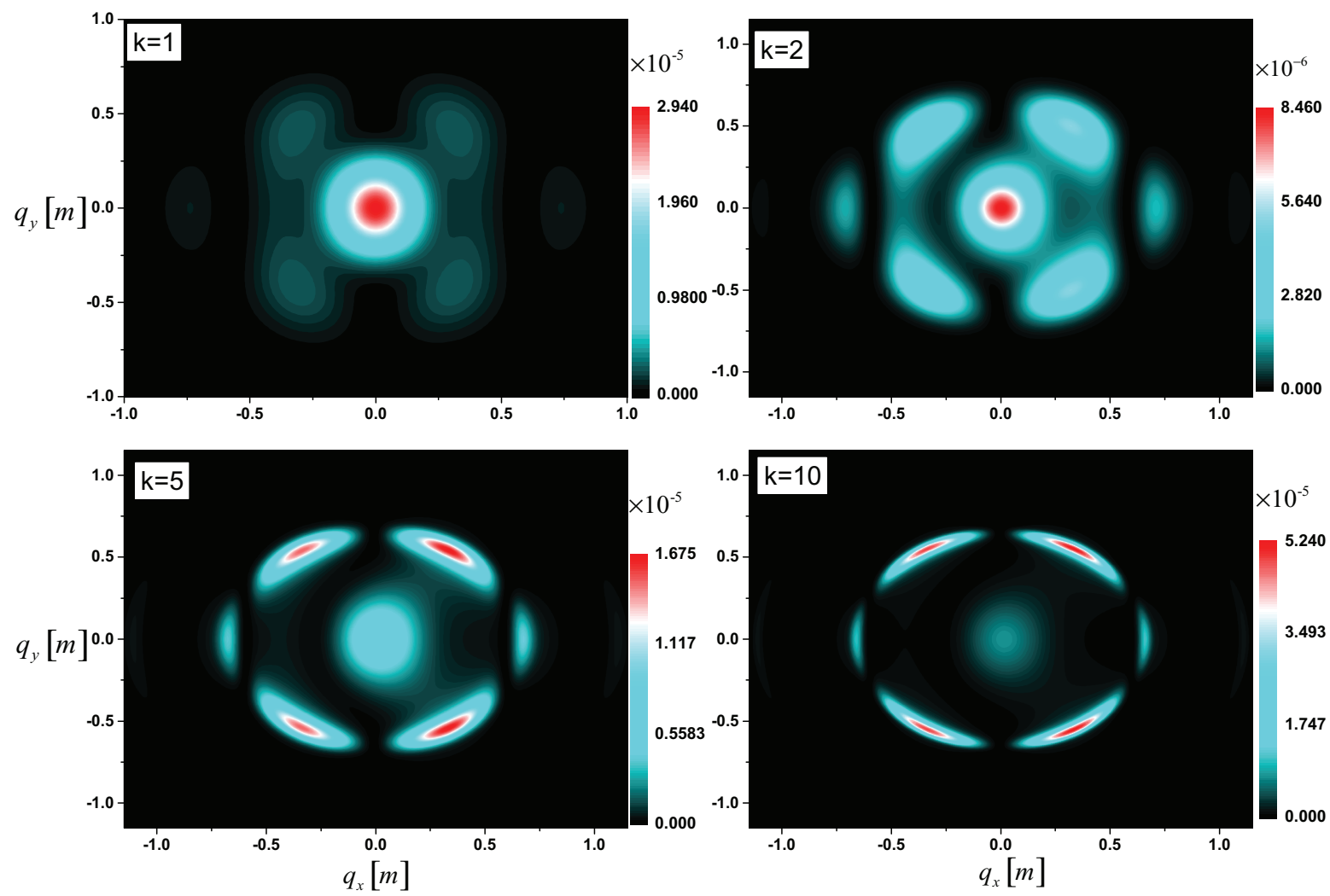

Figure 2. Same as Figure 1 except that the falling pulse length $\tau_{2}=k \tau_{1}$ becomes longer with $k \geq 1$.

appear and gradually become main peaks. This tendency is more striking with larger $k$.

We find that the center maximum value of the momentum spectrum decreases until $k \leq 5$. For the pulse length $k=10$, the maximum value at the ring is slightly larger than that of symmetrical pulse when $k=1$. Note that the ring structure in the momentum spectrum is a typical feature of the multiphoton pair production mechanism. Because the produced pairs momentum spectrum lines up exactly with the prediction that

$$
|\mathbf{q}|=\frac{1}{2} \sqrt{(n \omega)^{2}-\left(2 m_{*}\right)^{2}},
$$

after the considering of effective mass ${ }^{[23]}$, the Keldysh parameters are large enough to support multiphoton pair production. For example, the inner ring is formed by absorbing four photons and the outermost obscured structure corresponds to the absorption of five photons.

\subsection{Elliptic polarization, $\delta=0.5$}

For the middle-elliptical polarization case, $\delta=0.5$, in Figure 3 we show the momentum spectrum for shorter pulses. From the upper-left panel of Figure 3, where $k=1$, we see that the momentum spectrum is symmetrically distributed about the $q_{x}$ axis, and that the spectrum peak is located at $\mathbf{q}=\mathbf{0}$. With decreasing $k$, we observe that distortion of the momentum spectrum occurs, or, equivalently, the mirror symmetry about $q_{x}$ is lost. As the peak position shifts, the maximum value of the peak increases. For example, when $k=0.5$, the main peak shifts along the positive $q_{y}$ direction, while, when $k=0.3$, the main peak shifts along the negative $q_{x}$ direction with a slightly larger peak value. For the very asymmetric case of $k=0.1$, the momentum spectrum is concentrated around the center and the main peak is almost located at the center again.

Now let us consider the elongated falling pulse cases with $k \geq 1$ for the middle-elliptical polarization case, $\delta=0.5$; see Figure 4 . For $k=2$, the symmetry of the momentum spectrum about the $q_{x}$ axis is destroyed. The peak position shifts to the positive and negative $q_{y}$ direction, while the peak value decreases compared to the symmetric case, $k=1$. For larger $k$, the spectrum at the center vanishes gradually with increasing $k$, and a complete ringlike shape appears. The peak positions are very interesting which form two elongated strips by locating at the relative narrower regime of positive and negative $q_{y}$ but relative broader regime of positive and negative $q_{x}$. Finally, the additional outer ring structure again appears, which is a clear signal of multiphoton pair production processes. This can be understood from the fact that, with increasing pulse length $k \tau_{1}$, the electric field has long enough duration and changes its direction during the pair creation process. Thus, the created particles may be 

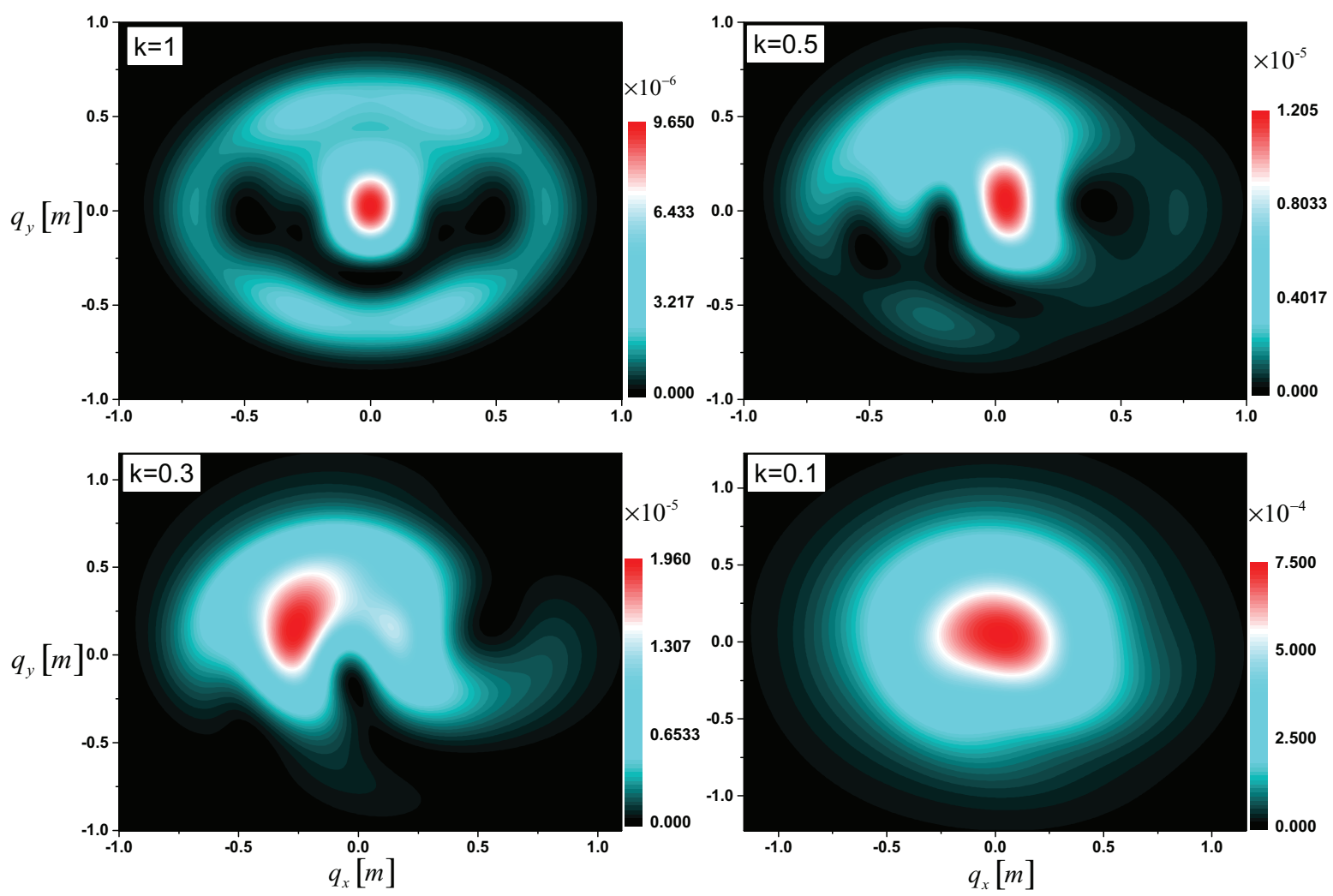

Figure 3. Same as Figure 1 except for elliptic polarization, $\delta=0.5$.
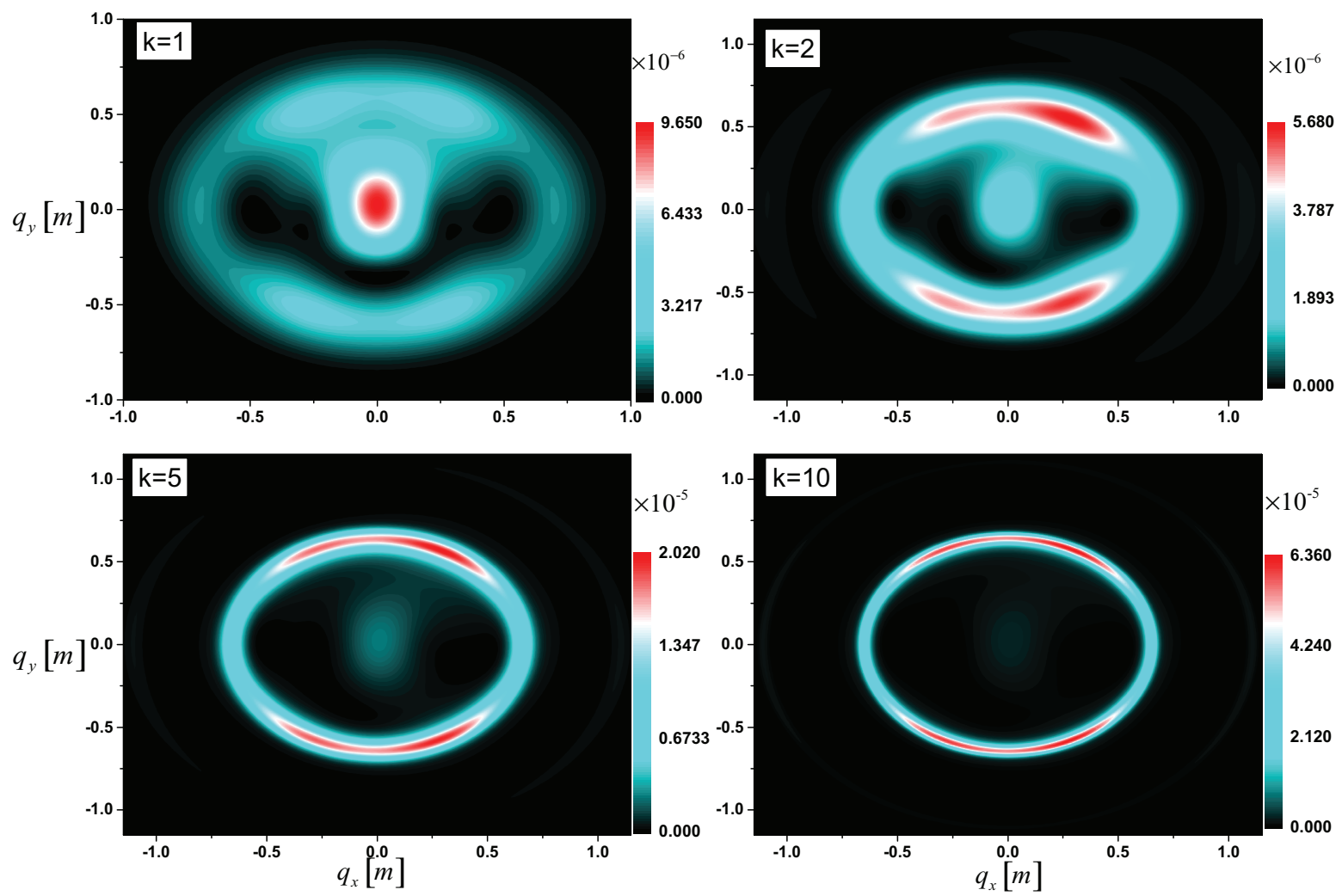

Figure 4. Same as Figure 2 except for elliptic polarization, $\delta=0.5$. 
accelerated in different directions depending on the field direction at the time of production. This results in a ring structure of the spectrum. On the other hand, as the pulse duration increases with $k$, the number of oscillation cycles within the Gaussian envelope also increases, and there will be more photons contributing to pair production by the multiphoton absorption mechanism, so in the spectrum the signal of multiphoton pair creation becomes pronounced with a ring shape. However, for the shorter pulse cases, the number of oscillation cycles in the envelope is very small, which does not show the complete multiphoton pair production signal in the momentum spectrum clearly since, for small pulse length $\tau$, the Keldysh parameter $\gamma=m \omega / e E_{0}$ will be influenced by the time scale $\tau$. This phenomenon also appears in the linear and circular polarizations.

\subsection{Circular polarization, $\delta=1$}

For the circular polarization, $\delta=1$, we show the momentum spectra in Figure 5 when the pulse length is shorter with $0<k \leq 1$. It can be seen that in the symmetric case, $k=1$, the momentum spectrum has an obvious ring structure centered around the origin, meanwhile a weak interference effect and/or oscillation is also observed between the hole and outer ring along the negative values of the $q_{y}$ axis; see Figure 5 for $k=1$. On the one hand, the ring shape arises from absorbing four photons in the multiphoton pair production. We know that the ring radius can be calculated using energy conservation, including the effective mass consideration, as $|\mathbf{q}|=\frac{1}{2} \sqrt{(n \omega)^{2}-\left(2 m^{*}\right)^{2}}$, where $n$ is the number of photons participating in the pair creation and $m^{*}$ is the effective mass ${ }^{[23]}$. On the other hand, the weak interference effect can be explained by analyzing the distribution of turning points in the semiclassical picture ${ }^{[33]}$. The complex-valued turning points are those $t_{p}$ that are obtained by $\Omega\left(q, t_{p}\right)=0$, which is responsible for the interference effects of the spectrum. We refer the reader to Ref. [33] for the interference pattern associated with the turning points.

With decreasing $k$, the peaks of the momentum spectra display quite a rich structure and the interference effects gradually vanish. When $k=0.7$, the peak appears in the upper-left side of the momentum spectrum space. When $k=0.3$, the partial ring structure vanishes and the momentum spectrum becomes distorted. For the very asymmetric case of $k=0.1$, the peak position is located at the near central region. Note that, for the circular polarization, the peak value of the momentum spectrum is remarkably enhanced by two orders of magnitude compared to that in the symmetric case, $k=1$.

We again consider the opposite situation of falling pulse change, i.e., the falling pulse length $\tau_{2}$ becomes longer with $k \geq 1$. The momentum spectra are shown in Figure 6. It is obvious that in this case the momentum distribution at the
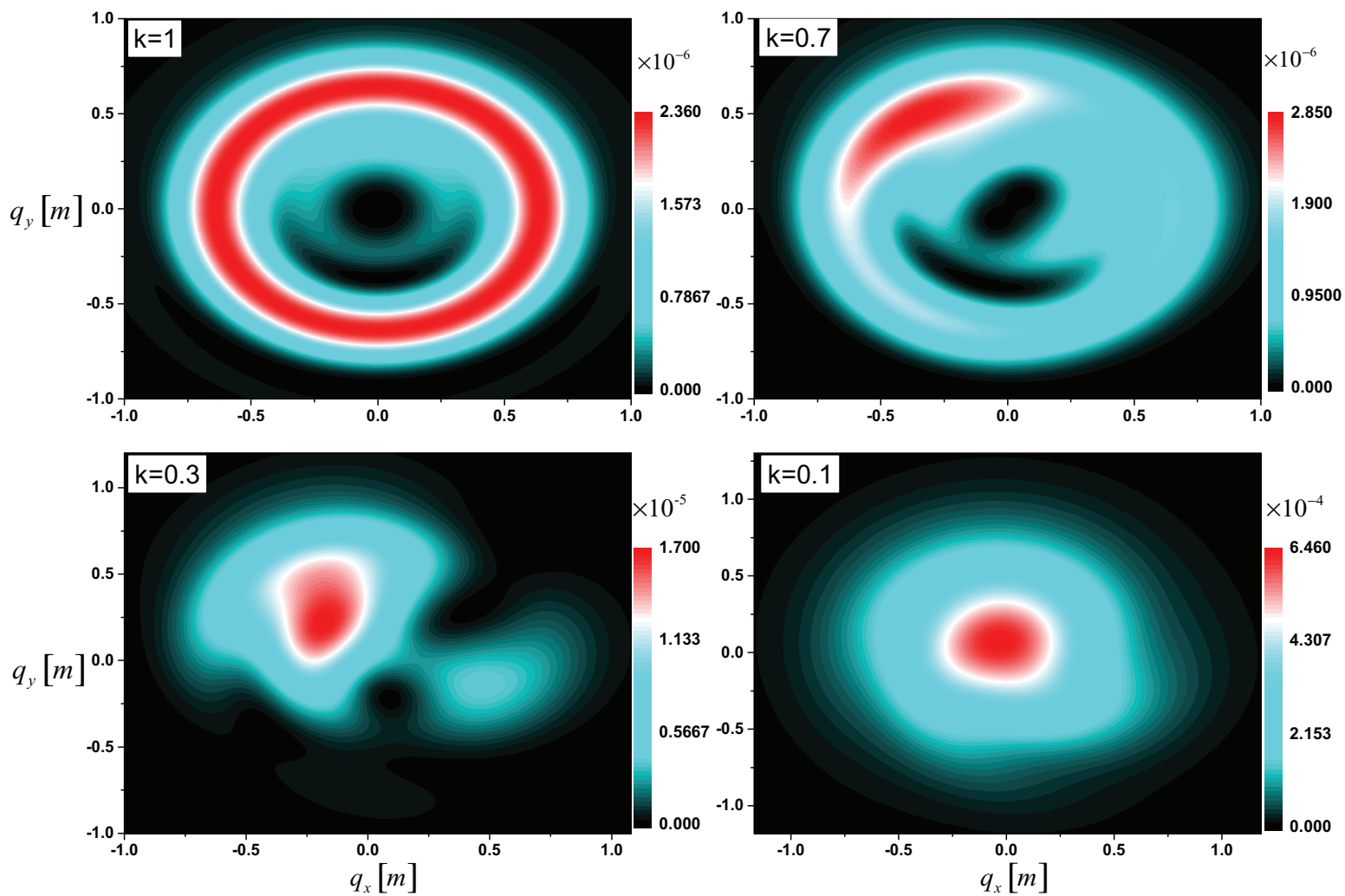

Figure 5. Same as Figure 1 except for circular polarization, $\delta=1$. 

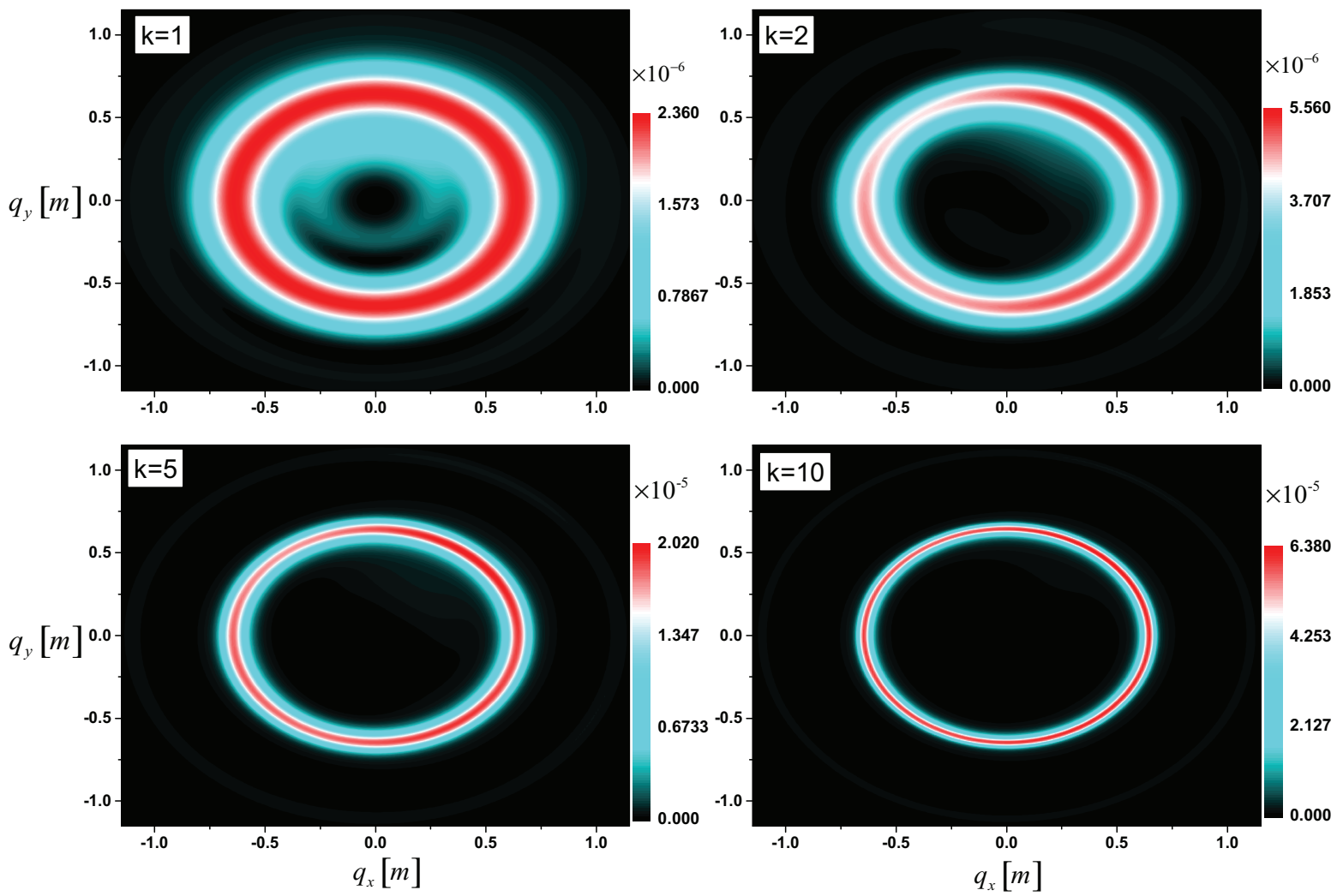

Figure 6. Same as Figure 2 except for circular polarization, $\delta=1$.

Table 1. The peak values of the particle distribution function $f(\mathbf{q}, \infty)$ for the typical polarization $\delta$ when the rising pulse length $\tau_{1}=10 / m$ is fixed and the falling pulse length $\tau_{2}=k \tau_{1}$ is short and/or elongated. Note that these peaks occur at different values of the momentum $\mathbf{q}$.

\begin{tabular}{lccc}
\hline & \multicolumn{3}{c}{$f_{\max }(\mathbf{q}, \infty)$ at peak } \\
\cline { 2 - 4 } & $k=1$ & $k=0.1$ & $k=10$ \\
\hline$\delta=0$ & $29.40 \times 10^{-6}$ & $8.28 \times 10^{-4}$ & $5.24 \times 10^{-5}$ \\
$\delta=0.5$ & $9.65 \times 10^{-6}$ & $7.50 \times 10^{-4}$ & $6.36 \times 10^{-5}$ \\
$\delta=1$ & $2.36 \times 10^{-6}$ & $6.46 \times 10^{-4}$ & $6.38 \times 10^{-5}$ \\
\hline
\end{tabular}

inner part of the ring gradually vanishes with increasing $k$, and that the red ring distribution becomes thin with lacking interference effect. Finally, the additional outer ring shape again appears, although it is a little obscure. The red inner ring in the momentum spectrum corresponds to the absorption of four photons; however, the outer ring corresponds to the absorption of five photons.

In Table 1 we list some corresponding peak values of the momentum distribution for different polarizations. We find that in the shorter falling pulse cases the peak value of the momentum spectrum is enhanced but this enhancement decreases as the field polarization increases. In the elongated falling pulse cases the peak value is also enhanced. However, on the one hand, this enhancement increases as the field polarization increases, while, on the other hand, the enhancements in the elongated cases are weaker globally compared to the shorter pulse cases.

\section{Number density of pair production}

In this section we calculate the change of the pair number density generated in different polarization electric fields with asymmetric shape and different pulse length ratio $k$. The results are shown in Figures 7 and 8 for shorter and elongated falling pulses, respectively.

We find that, when the falling pulse width is shorter, i.e., $0<k \leq 1$, the number density of created pairs decreases with the increasing electric field polarization. We also find that the number density of $e^{-} e^{+}$pairs in different polarizations increases with decreasing pulse length ratio value $k$. For the larger compression, it is more obvious, especially for $k=0.4$ to $k=0.1$. When the pulse length is shorter, the number density increases by more than two orders of magnitude for each polarization. As $k$ decreases, the electric field comprises a strong pulse (when $t<0$ ) superimposed with a weak pulse (when $t>0$, but having wider frequency components in the sense of Fourier decomposition); therefore, these two half pulses with different time scales act as an effective dynamically assisted mechanism. Thus, this results in an enhancement of the number density of produced pairs.

Concretely, for linear polarization, the number density increases from $1.20 \times 10^{-7}$ when $k=1$ to $1.853 \times 10^{-5}$ 


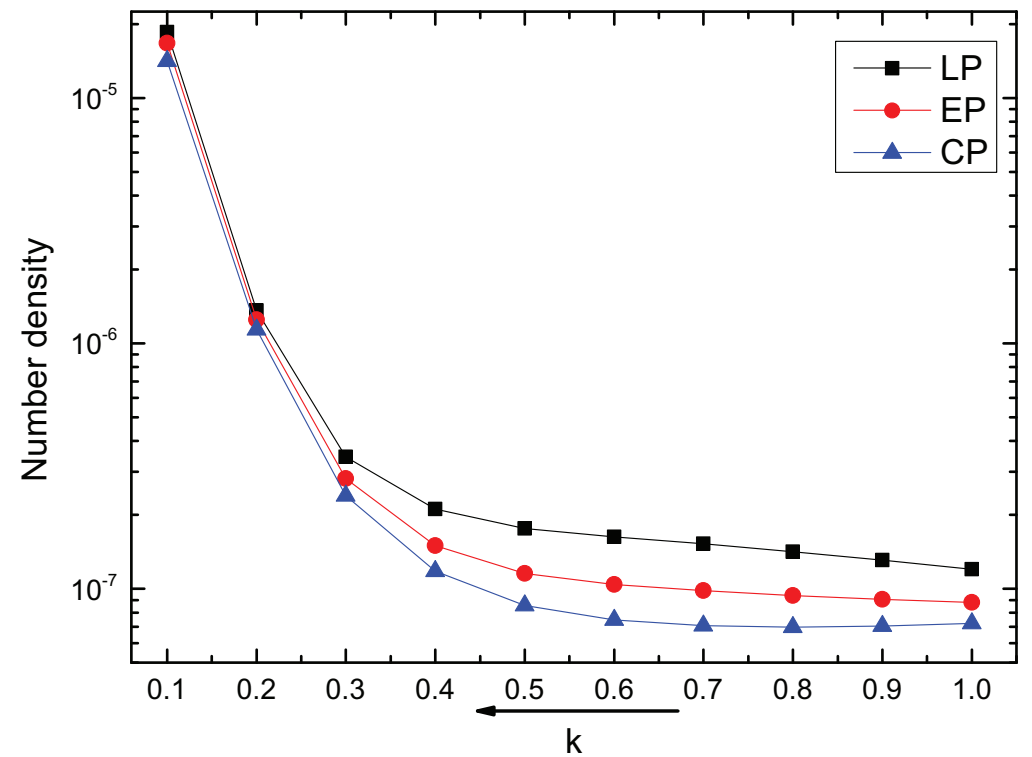

Figure 7. The number density (in units of $\lambda_{c}^{-3}=m^{3}$ ) of pairs produced in differently polarized electric fields for the shorter falling length of the asymmetric pulse shape with $0<k \leq 1$. The field parameters are the same as in Figure 1. Here LP, EP and CP with squares, circles and triangles denote the linear $\delta=0$, elliptical $\delta=0.5$ and circular $\delta=1$ cases, respectively.

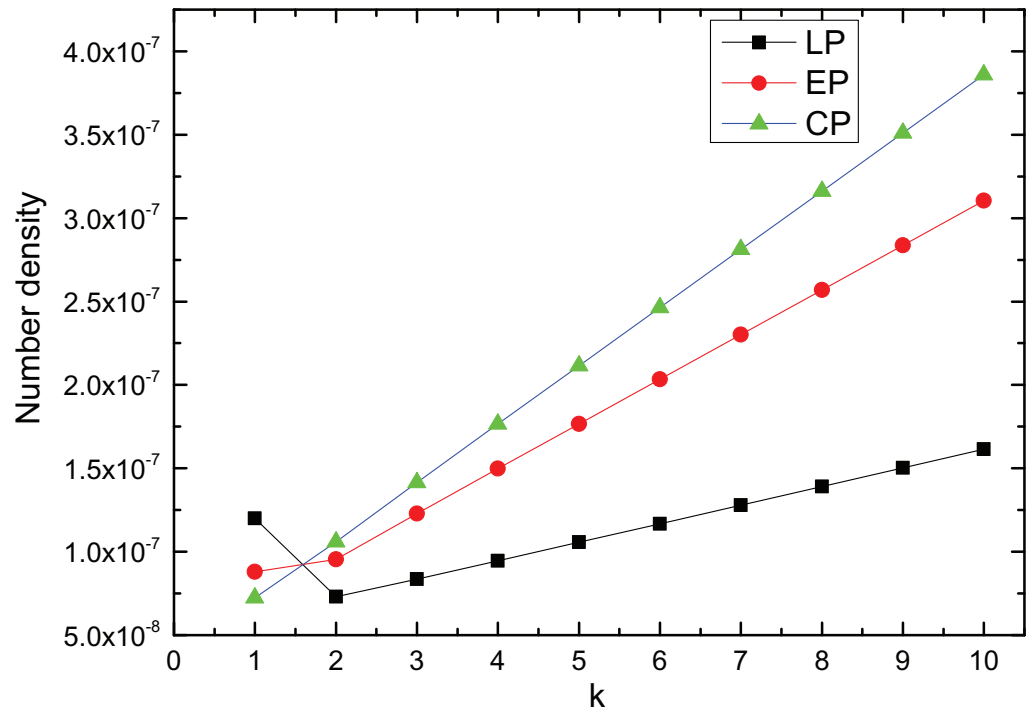

Figure 8. Same as Figure 7 except for the elongated falling case with $k \geq 1$.

when $k=0.1$. For elliptical polarization, it increases from $8.799 \times 10^{-8}$ when $k=1$ to $1.673 \times 10^{-5}$ when $k=0.1$. For circle polarization, it increases from $7.237 \times 10^{-8}$ when $k=1$ to $1.414 \times 10^{-5}$ when $k=0.1$.

On the other hand, in the case of the elongated falling pulse, the number density of created pairs increases almost linearly with the field polarization parameter $\delta$ as well as the pulse elongation parameter $k$ except that for linear polarization, it decreases little when the falling pulse elongation is not large, but still increases with larger $k$. This is mainly attributed to effect of field asymmetry due to the puse length on the pair production processes. These results therefore show that the degree of pulse asymmetry is an important parameter in the pair production process for polarized electric fields. Note that our results are similar to the findings of Kohlfürst et al. ${ }^{[24]}$, who considered the single Sauter pulse. They found that the particle number increases first with increasing pulse length until it reaches $\tau=0.5 \mathrm{~m}^{-1}$, then decreases and reaches its minimum at $\tau=30 \mathrm{~m}^{-1}$ and finally increases again slowly; see Figure 4 of Ref. [24].

From Figures 7 and 8, we can infer that the number density exhibits polarization dependence for shorter pulse asymmetry and elongated pulse asymmetry of the field. For shorter pulse asymmetry cases, the number density decreases with increasing field polarization, while for elongated pulse cases, the number density increases with increasing field 
polarization except for the case in which $k=1$. However, the number density has a minimum at some $k$ for each polarization field, for example, $k=2$ and $k=0.8$ correspond to linear $(\delta=0)$ and circular $(\delta=1)$ polarization fields, respectively. The number density exhibiting this nonlinear behavior with $k$ can be attributed to the following two features. For larger $k$, because the duration is elongated, the number increases almost linearly with $k$ for an almost constant pair production rate. On the other hand, for progressively smaller $k$, progressively wider frequencies make the dynamically assisted mechanism progressively more effective so that it enhances the number density.

In summary, when the falling pulse length is shortened, the number density can be increased by two orders of magnitude; however, when the falling pulse length is extended, the number density is enhanced to within only half orders of magnitude. Therefore, for asymmetric electric fields with different polarizations, in order to effectively increase the number density of the produced electron-positron pairs, it is better to shorten the falling pulse. Note that in our previous work on the linearly polarized case ${ }^{[25]}$, in which we used the quantum Vlasov equation approach, a similar finding was qualitatively presented.

\section{Summary and discussion}

In this study we have investigated the effects of the asymmetric pulse shape on the momentum spectrum of created $e^{-} e^{+}$ pairs in strong electric fields for three different polarization fields, linearly, middle elliptically and circularly polarized fields, on the momentum spectrum of created particles by applying the DHW formalism. The main results for the spectra of produced pairs can be summarized as follows.

When the falling pulse length is shorter, for linear polarization, the spectra of the produced pairs exhibit a shift and split of the peaks. For middle elliptic polarization as well as circular polarization, the momentum spectra become distorted and exhibit a shift of the peaks. Finally, for each different polarization, the peaks shifted to the central region in the momentum plane; therefore, peak values were enhanced by two orders of magnitude compared to the symmetric situation. When the falling pulse length is elongated, ring structures appear for different polarizations. We also noted that, for this asymmetric situation, the peak values increased with the field polarization compared to the symmetric case, but were smaller than in the shorter pulse cases. Some phenomena of the momentum spectra are consistent with the effect of frequency chirp ${ }^{[33]}$.

We also studied the effect of asymmetric falling pulse on the obtained number density. We found that the number density decreases and/or increases with polarization for shorter and/or elongated falling pulses. It is important to note that, when the falling pulse is shorter, the number density of the produced pairs can be significantly enhanced by more than two orders of magnitude.

The results are helpful to understand the influence of the pulse duration, which is an important parameter of the external field, and to deepen our understanding of the external pulse structure. Although these results reveal some useful information about the production of $e^{+} e^{-}$pairs in different elliptical polarization cases, in this study we restricted ourselves to multiphoton pair creation, so asymmetric pulse shape effects for pair creation under the Schwinger mechanism need to be studied further for different polarized fields.

To understand why the multiphoton process is not obvious for shorter pulse cases $k<1$, we note that the traditional standard multiphoton pair production is weaker because the Keldysh parameter $\gamma=m \omega / e E_{0}$ is modified by the inverse of another time scale $\tau$ of the pulse duration. For very small $\tau$, this means that the oscillation number of the field includes fewer cycles and/or subcycles so that it is strictly not a complete multiphoton process. However, in this case, the number density of pairs can be increased remarkably due to the dynamically assisted mechanism. On the other hand, for the elongation pulse $k>1$, as $k$ increases, pair creation is dominated by the multiphoton mechanism; at this time for $\omega=0.6 m$, the corresponding number density for the circular polarization is greater than that for the middle elliptical polarization, with the latter greater than that for the linear polarization case (see also Figure 4 of Ref. [31]).

The other important phenomenon observed in our numerical results is the spiral structure in the momentum spectrum that has an intrinsic connection with the spin and/or orbital angular momentum of field photons as well as the produced $e^{-} e^{+}$particles. The theoretical analysis for this characteristic is not easy and almost completely ignored in the present study. However, its abundant information about the rotation degree is very important and helpful in understanding the involved strong external field interaction with a vacuum and possible applications in future real experiments.

\section{Acknowledgments}

The authors are thankful to the anonymous referees for their helpful suggestions to improve the presentation of the paper. The work of O. Olugh and B.-S. Xie was supported by the National Natural Science Foundation of China (NSFC) under Grant Nos. 11875007 and 11935008 . The work of Z.-L. Li was supported by the NSFC under Grant No. 11705278. The computation was carried out at the HSCC of the Beijing Normal University.

\section{References}

1. F. Sauter, Z. Phys. 69, 742 (1931).

2. W. Heisenberg and H. Euler, Z. Phys. 98, 714 (1936).

3. J. S. Schwinger, Phys. Rev. 82, 664 (1951).

4. F. Gelis and N. Tanji, Prog. Part. Nucl. Phys. 87, 1 (2016). 
5. T. Heinzl and A. Ilderton, Eur. Phys. J. D 55, 359 (2009).

6. M. Marklund and J. Lundin, Eur. Phys. J. D 55, 319 (2009).

7. O. J. Pike, F. Mackenroth, E. G. Hill, and S. J. Rose, Nature Photon. 8, 434 (2014).

8. A. Ringwald, Phys. Lett. B 510, 107 (2001).

9. R. Alkofer, M. B. Hecht, C. D. Roberts, S. M. Schmidt, and D. V. Vinnik, Phys. Rev. Lett. 87, 193902 (2001).

10. C. D. Roberts, S. M. Schmidt, and D. V. Vinnik, Phys. Rev. Lett. 89, 153901 (2002).

11. M. Orthaber, F. Hebenstreit, and R. Alkofer, Phys. Lett. B 80, 698 (2011)

12. D. Vasak, M. Gyulassy, and H. T. Elze, Annals Phys. 173, 462 (1987).

13. I. Bialynicki-Birula, P. Górnicki, and J. Rafelski, Phys. Rev. D 44, 1825 (1991).

14. F. Hebenstreit, R. Alkofer, and H. Gies, Phys. Rev. D 82, 105026 (2010).

15. F. Hebenstreit, R. Alkofer, and H. Gies, Phys. Rev. Lett. 107, 180403 (2011).

16. F. Hebenstreit, arXiv:1106.5965 (2011).

17. C. Kohlfürst, arXiv:1512.06082 (2015).

18. C. Kohlfürst, Eur. Phys. J. Plus 133, 191 (2018)

19. E. Akkermans and G. V. Dunne, Phys. Rev. Lett. 108, 030401 (2012).
20. C. K. Dumlu and G. V. Dunne, Phys. Rev. Lett. 104, 250402 (2010).

21. R. Schützhold, H. Gies, and G. Dunne, Phys. Rev. Lett. 101, 130404 (2008).

22. F. Hebenstreit, R. Alkofer, G. V. Dunne, and H. Gies, Phys. Rev. Lett. 102, 150404 (2009).

23. C. Kohlfürst, H. Gies, and R. Alkofer, Phys. Rev. Lett. 112, 050402 (2014).

24. C. Kohlfürst, M. Mitter, G. von Winckel, F. Hebenstreit, and R. Alkofer, Phys. Rev. D 88, 045028 (2013).

25. O. Oluk, B. S. Xie, M. Bake, and S. Dulat, Front. Phys. 9, 157 (2014).

26. I. A. Aleksandrov, G. Plunien, and V. M. Shabaev, Phys. Rev. D 95, 056013 (2017).

27. B. S. Xie, Z. L. Li, and S. Tang, Matter Radiat. Extremes 2 , 225 (2017).

28. A. Blinne and H. Gies, Phys. Rev. D 89, 085001 (2014).

29. A. Blinne and E. Strobel, Phys. Rev. D 93, 025014 (2016).

30. A. Blinne, arXiv:1701.00743 (2016).

31. Z. L. Li, D. Lu, and B. S. Xie, Phys. Rev. D 92, 085001 (2015).

32. O. Olugh, Z. L. Li, and B. S. Xie, Phys. Lett. B 802, 135259 (2020).

33. O. Olugh, Z. L. Li, B. S. Xie, and R. Alkofer, Phys. Rev. D 99, 036003 (2019). 